\title{
On the Coordinatization of Oriented Matroids
}

\author{
Jürgen Bokowski and Bernd Sturmfels \\ Technische Hochschule Darmstadt, Fachbereich Mathematik, Schlossgartenstrasse 7, \\ 6100 Darmstadt, West Germany
}

\begin{abstract}
Several important and hard realizability problems of combinatorial geometry can be reduced to the realizability problem of oriented matroids. In this paper we describe a method to find a coordinatization for a large class of realizable cases. This algorithm has been used successfully to decide several geometric realizability problems. It is shown that all realizations found by our algorithm fulfill the isotopy property.
\end{abstract}

\section{Introduction}

Several important and hard realizability problems of combinatorial geometry can be reduced to the realizability problem of oriented matroids. Thus the polytopality or the star-shape embeddability of combinatorial spheres, or more generally, the geometric embeddability of abstract complexes can be transformed by discrete algorithms to the problem of finding a coordinatization of at least one corresponding oriented matroid, see, e.g., [9].

Nevertheless, to decide whether a given oriented matroid is realizable is still a hard problem and no efficient algorithm is known to answer this question for the general case. On the other hand, by a very general theorem of Tarski [18] the decidability of this problem can be concluded. Using the special structure of inequality systems arising from chirotopes [8] as signed bases representation for oriented matroids, we propose a method to find a coordinatization for a large class of realizable cases.

This algorithm has been used successfully to decide several geometric realizability problems, [5], [7], the completion of the enumeration of all neighborly 4-polytopes with ten vertices [9] and an embedding of a 2-manifold of genus 3 with ten vertices in 3-space have been achieved by this method [4].

In the first two sections we sketch the basic notions of chirotope theory. The realizability problem for $d$-chirotopes with $n$ vertices is seen to be equivalent to the existence of a real-valued $(n-d) \times d$-matrix for which the signs of all subdeter- 
minants are prescribed. These inequality systems have a very special structure, which allows one to describe the algebraic solution process in purely combinatorial terms. Among this combinatorial calculus, developed in Section 4, the differentiation rule in Theorem 2 might be of interest on its own.

The concept of solvability sequences for chirotopes is the main topic of this paper. A solvability sequence is, roughly speaking, an ordering of the variables that allows the choice of coordinates in a nonprospective way. It is shown that the class of realizable chirotopes with solvability sequences is quite rich.

Several well-known construction techniques from oriented matroid theory and convexity are special cases of this method. Although it is very likely that configurations without solvability sequences do exist, so far no examples are known to the authors."

The last section relates the realization problem and the isotopy conjecture for nondegenerate configurations posed by Goodman and Pollack [18]. It is proved that configurations with solvability sequences do have the isotopy property and even more: the configuration space turns out to be not only connected, but contractible.

\section{Realizability of Chirotopes}

We assume that the reader is familiar with the basic concepts of oriented matroid theory and Grassmann algebra: see [8] and references cited in this paper. Throughout our paper we use chirotopes, as defined below, as representations of oriented matroids. For equivalence proofs between the chirotope and the classical definition of an oriented matroid given in [3] see Las Vergnas [15], [16] (one direction), Lawrence [17], Dress (letter to Las Vergnas 1982) and [8].

We give some notations. Let

$$
\Lambda(n, d):=\left\{\left(\lambda_{1}, \ldots, \lambda_{d}\right) \in N^{d}: 1 \leq \lambda_{1}<\cdots<\lambda_{d} \leq n\right\} .
$$

Definition 2.1. A mapping $\chi: \Lambda(n, d) \rightarrow\{+1,-1,0\}$ (alternatingly extended to all $d$-tuples of $\left.\{1,2, \ldots, n\}^{d}\right)$ is called a chirotope if for all $\sigma \in \Lambda(n, d-2), \tau \in \Lambda(n, 4)$ the set

$$
\begin{gathered}
\left\{\chi\left(\sigma_{1}, \ldots, \sigma_{d-2}, \tau_{1}, \tau_{2}\right) \cdot \chi\left(\sigma_{1}, \ldots, \sigma_{d-2}, \tau_{3}, \tau_{4}\right),\right. \\
-\chi\left(\sigma_{1}, \ldots, \sigma_{d-2}, \tau_{1}, \tau_{3}\right) \cdot \chi\left(\sigma_{1}, \ldots, \sigma_{d-2}, \tau_{2}, \tau_{4}\right), \\
\left.\chi\left(\sigma_{1}, \ldots, \sigma_{d-2}, \tau_{1}, \tau_{4}\right) \cdot \chi\left(\sigma_{1}, \ldots, \sigma_{d-2}, \tau_{2}, \tau_{3}\right)\right\}
\end{gathered}
$$

either contains $\{-1,+1\}$ or equals $\{0\}$.

$\chi$ is called realizable if $\chi=\operatorname{sign}\left(\bigwedge_{d} M\right.$ ) for a real-valued $n \times d$-matrix $M$, where $\bigwedge_{d} M$ denotes the $d$ th compound matrix of $M$, i.e., the $d$-vector of all $\left(\begin{array}{l}n \\ d\end{array}\right)$ $d \times d$-subdeterminants of $M$.

Given a subset $X=\left\{x_{1}, \ldots, x_{n}\right\} \subset R^{d}$ we associate with $X$ the realizable chirotope $X=\operatorname{sign} \bigwedge_{d}\left(x_{1}, \ldots, x_{n}\right)^{t}$ which assigns to each basis in $X$ its orientation.

\footnotetext{
In the meantime, Jürgen Richter from Darmstadt gave an example with 13 points in the plane.
} 


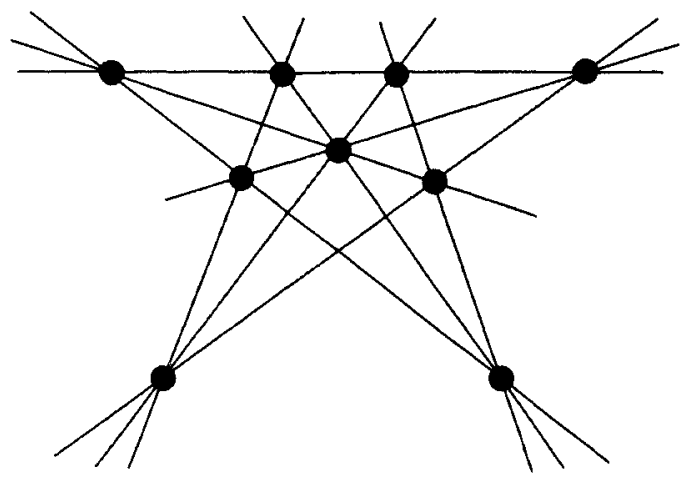

Fig. 1

If $x_{i}=\left(1, y_{i}\right), i=1, \ldots, n$, then $\chi$ assigns to each $d$-simplex in $Y=$ $\left\{y_{1}, \ldots, y_{n}\right\} \subset R^{d-1}$ its orientation. Interpreting every chirotope $\chi$ this way, we call $d$ the rank and $n$ the number of vertices of $\chi \cdot \chi$ is a simplicial chirotope, if $\operatorname{Im} \chi \subset\{-1,+1\}$.

In the following we consider mainly simplicial chirotopes, in which case chirotopes are oriented matroids. In the nonsimplicial case this is true only if the family $B_{\chi}$ of subsets $\left\{\lambda_{1}, \ldots, \lambda_{d}\right\} \subset\{1, \ldots, n\}$ with $\chi\left(\lambda_{1}, \ldots, \lambda_{d}\right) \neq 0$ forms the set of bases of a matroid.

It is known [8] that there exists an infinite family of minorminimal nonrealizable simplicial $d$-chirotopes for $d \geq 3$. Hence an easy combinatorial realizability criterion by excluding a finite number of subchirotopes cannot exist. Furthermore, it is known that realizability depends on the field. For example, the 3-chirotope of simplex orientations of the following configuration with nine vertices is realizable with real numbers but not with rationals [13].

Clearly, real realizable simplicial chirotopes are also realizable with rationals and therefore also with integers. We restrict ourselves to simplicial chirotopes. The reader will see that all ideas can principally be extended to nonsimplicial chirotopes, too.

\section{Inequality Systems Arising from Chirotopes}

The question, whether a given simplicial $d$-chirotope $\chi$ with $n$ vertices is realizable leads immediately to a system of $\left(\begin{array}{c}n \\ d\end{array}\right) d \times d$-determinant inequalities in $n \cdot d$ variables. Since $\bigwedge_{d}(M \cdot A)=\bigwedge_{d} M \cdot \operatorname{det} A$ for every $d \times d$-matrix $A, d^{2}$ of these variables are redundant. We describe how the above inequality system can be transformed into a more convenient system in $(n-d) \times d$ variables.

If $\chi(\beta)=1$ for $\beta \in \Lambda(n, d)$ we can assume that the $n \times d$-realization matrix $V=\left(v_{k l}\right)$, i.e., $\operatorname{sign} \bigwedge_{d} V=\chi$, has the unit matrix as submatrix: $v_{\beta_{i, j}}=\delta_{i, j}$ $(1 \leq i, j \leq d)$. 
For $\lambda \in \Lambda(n, d)$ we use as notation for the determinant

$$
\operatorname{det}\left(v_{\lambda_{i, j}}\right)_{1 \leq i, j \leq d}=:\left(\lambda_{1}, \ldots, \lambda_{d}\right)=:(\lambda) \text {. }
$$

We have

$$
\beta\left[\beta_{j} \mid k\right]:=\left(\beta_{1} \cdots \beta_{j-1} k \beta_{j+1} \cdots \beta_{d}\right)=v_{k, j}, \quad k \notin \beta, \quad 1 \leq j \leq d .
$$

Similarly every $d \times d$-subdeterminant $(\lambda)$ of $V$, except $(\beta)$, corresponds to an $r \times r$-subdeterminant of the $(n-d) \times d$-matrix $\left(v_{k, j} ; k \notin \beta\right)$ where $1 \leq r:=|\lambda \backslash \beta| \leq$ $\min \{n-d, d\}$.

Example 3.1. $n=6, d=3, \beta=(235)$

$$
\begin{aligned}
V & =\left|\begin{array}{ccc}
v_{11} & v_{12} & v_{13} \\
1 & 0 & 0 \\
0 & 1 & 0 \\
v_{41} & v_{42} & v_{43} \\
0 & 0 & 1 \\
v_{61} & v_{62} & v_{63}
\end{array}\right|, \\
(245)=\beta\left[\beta_{2} \mid 4\right] & =\left|\begin{array}{ccc}
1 & 0 & 0 \\
v_{41} & v_{42} & v_{43} \\
0 & 0 & 1
\end{array}\right|=v_{42}, \\
(246) & =\left|\begin{array}{ccc}
1 & 0 & 0 \\
v_{41} & v_{42} & v_{43} \\
v_{61} & v_{62} & v_{63}
\end{array}\right|=\left|\begin{array}{ll}
v_{42} & v_{43} \\
v_{62} & v_{63}
\end{array}\right|, \\
\tau & =|\{2,4,6\} \backslash\{2,3,5\}|=2 .
\end{aligned}
$$

This correspondence is described by the following theorem.

Theorem 1. Let $M$ be an $n \times d$-matrix $(n \geq d)$ with $(\beta) \neq 0$. Then for all $\lambda \in \Lambda(n, d)$ :

$$
(\lambda) \cdot(\beta)^{d-1}=\operatorname{det}\left(\beta\left[\beta_{i} \mid \lambda_{j}\right]\right)_{1 \leq i, j \leq d} .
$$

The proof follows directly by multiplying $M=\left(m_{i j}\right)$ with the adjoint of $\left(m_{\beta_{i, j}}\right)_{1 \leq i, j \leq d}$.

Example 3.2. With Example 3.1 we have for any $6 \times 3$-matrix $M$;

$$
\begin{aligned}
(146)(235)^{2} & =\left|\begin{array}{lll}
(235)[2 \mid 1] & (235)[3 \mid 1] & (235)[5 \mid 1] \\
(235)[2 \mid 4] & (235)[3 \mid 4] & (235)[5 \mid 4] \\
(235)[2 \mid 6] & (235)[3 \mid 6] & (235)[5 \mid 6]
\end{array}\right| \\
& =\left|\begin{array}{lll}
(135) & (215) & (231) \\
(435) & (245) & (234) \\
(635) & (265) & (236)
\end{array}\right|
\end{aligned}
$$


It can be seen that all quadratic Grassmann-Plücker-relations, see [8], are special cases of (1).

Now consider a simplicial $d$-chirotope $\chi$ with $n$ vertices and $\chi(\beta)=+1$, which can be assumed for every $\beta \in \Lambda(n, d)$ in the simplicial case. By Theorem 1 each $\Delta \in \Lambda(n, d) \backslash\{\beta\}$ can be identified with a determinant function $\Delta\left(v_{1}, \ldots, v_{(n-d) d}\right)$ where

$$
\begin{aligned}
V_{\beta}:=\left\{v_{1}, \ldots, v_{(n-d) d}\right\}: & =\{\lambda \in \Lambda(n, d):|\lambda \backslash \beta|=1\} \\
& =\left\{\beta\left[\beta_{j} \mid k\right]: 1 \leq j \leq n, k \notin \beta\right\}
\end{aligned}
$$

is the set of independent variables. The sign matrix $\left(\chi\left(\beta\left[\beta_{j} \mid k\right]\right): k \notin \beta\right)$ is known in oriented matroid theory as standard representative matrix according to a basis $\beta \in \Lambda(n, d)$, see [2]. The realizability question for $\chi$ can be posed in the following form:

Do there exist real numbers $\eta_{1}, \ldots, \eta_{(n-d) d} \in R$ such that

$$
\operatorname{sign} \Delta_{i}\left(\ldots, \eta_{j}, \ldots\right)=\chi\left(\Delta_{i}\right) \text { for all } \Delta_{i} \in \Lambda(n, d) \backslash\{\beta\} ?
$$

Solving such a system of $\left(\begin{array}{c}n \\ d\end{array}\right)-1$ simultaneous inequalities successively by eliminating the variables $v_{i}$, we are still free

to choose a suitable basis $\beta$,

to choose an elimination order, solvability sequence,

to reduce the system by the chirotope conditions.

Definition 3.3. $R \subset \Lambda(n, d)$ is called a reduced system for $\chi$, if for every chirotope $\chi^{\prime}$ with $\left.\chi^{\prime}\right|_{R}=\left.\chi\right|_{R}$ we have $\chi^{\prime}=\chi$. A minimal (with respect to inclusion) reduced system will be called a minimal system for $\chi$. Obviously (2) is equivalent to

Given a minimal system $M \subset \Lambda(n, d)$, do there exist $\eta_{1}, \ldots, \eta_{(n-d) d} \in R$ such that

$$
\operatorname{sign} \Delta_{i}\left(\ldots, \eta_{j}, \ldots\right)=\chi\left(\Delta_{i}\right) \text { for all } \Delta_{i} \in M ?
$$

If we want to eliminate a variable $v_{1}$ in some minimal inequality system $\left\{\Delta_{i}<0(>0)\right\}$, we write

$$
\Delta_{i}\left(v_{1}, v_{2}, \ldots\right)=v_{1} \cdot \Delta_{i}^{1}\left(v_{2}, \ldots\right)+R_{i}\left(v_{2}, \ldots\right),
$$

where $R_{i}$ and $\Delta_{i}^{1}=\partial \Delta_{i} / \partial v_{1}$ are homogeneous polynomials in $v_{2}, v_{3}, \ldots$ Since $\Delta_{i}^{1}$ is again a determinant expression arising from $\Lambda(n, d)$ and $\chi\left(\Delta_{i}^{1}\right) \neq 0$ by assumption, we get one of the following inequalities for $v_{1}$ :

(i) $v_{1}<-R_{i} / \Delta_{i}^{1}\left(v_{2}, \ldots\right)$;

(ii) $v_{1}>-R_{j} / \Delta_{j}^{1}\left(v_{2}, \ldots\right)$. 
With this partition of all determinants $\Delta_{i}$ containing $v_{i}$ in upper bounds (i) and lower bounds (ii) in general we obtain new inequalities

(iii) $-\boldsymbol{R}_{j} / \Delta_{j}^{1}\left(v_{2}, \ldots\right)<-\boldsymbol{R}_{\mathbf{i}} / \Delta_{i}^{1}\left(v_{2}, \ldots\right)$

for all upper bounds $\Delta_{i}$ and lower bounds $\Delta_{j}$. In the case of only one-sided restrictions for $v_{1}$ no new inequalities (iii) occur.

These new polynomial inequalities

(iv) $P_{i j}:=R_{j} D_{i}^{1}-R_{i} D_{j}^{1}>0(<0)$ together with the $\Delta_{k}$ not containing $v_{1}$ form a new inequality system, such that the old system has a solution $v_{1}, v_{2}$, $v_{3}, \ldots$ if and only if the new system has a solution $v_{2}, v_{3}, \ldots$

Since the $\boldsymbol{P}_{i j}$ are polynomials of higher degree, in general it will be impossible to continue this elimination successively. This is the crucial point why a general algorithm is not reached in this way. Therefore we restrict ourselves to the cases where either no new polynomials $P_{i j}$ occur or all new polynomials are of the form $P_{i j}= \pm \Delta_{k} \cdot \Delta_{l}$ for suitable $\Delta_{k}, \Delta_{l} \in \Lambda(n, d)$.

Example 3.4 (compare Example 3.1 with $(\beta)=(235)$ ). The following reduced system defines a chirotope $\chi$ :

Table 1

\begin{tabular}{llll}
\hline $123+$ & $125+$ & $135+$ & $136+$ \\
$146+$ & $156+$ & $234+$ & $235+$ \\
$236+$ & $245-$ & $246-$ & $256+$ \\
$345-$ & $346+$ & $356+$ & $456-$ \\
\hline
\end{tabular}

With $v_{1}:=(123)=v_{13}$,

$$
\begin{aligned}
\Delta_{i} & :=\left|\begin{array}{ll}
v_{11} & v_{13} \\
v_{61} & v_{63}
\end{array}\right| \text { and } \\
\Delta_{j}: & =\left|\begin{array}{lll}
v_{11} & v_{12} & v_{13} \\
v_{41} & v_{42} & v_{43} \\
v_{61} & v_{62} & v_{63}
\end{array}\right|,
\end{aligned}
$$

we obtain

$$
\Delta_{i}^{1}=-v_{61} \text { and } \quad \Delta_{j}^{k}=\left|\begin{array}{ll}
v_{41} & v_{42} \\
v_{61} & v_{62}
\end{array}\right|
$$

and therewith

$$
R_{j} \cdot \Delta_{i}^{1}-R_{i} \cdot \Delta_{j}^{1}=-\left|\begin{array}{ll}
v_{11} & v_{12} \\
v_{61} & v_{62}
\end{array}\right| \cdot\left|\begin{array}{ll}
v_{41} & v_{43} \\
v_{61} & v_{63}
\end{array}\right|=-(156) \cdot(346)<0 .
$$

Elimination of $v_{1}$ yields the new inequality system in which (123), (136), and (146) do not occur. In the next section we develop a combinatorial calculus to describe and compute effectively eliminations of this type. 


\section{The Combinatorial Calculus}

Using the terminology of the last chapter, we begin with a theorem that describes the relationship between $\Delta_{i} \in \Lambda(n, d)$ and its derivatives $\Delta_{i}^{j}=\partial \Delta_{i} / \partial v_{j} \in \Lambda(n, d)$.

Theorem 2. Interpreting $\Delta \in \Lambda(n, d)$ as a polynomial depending on the variables $V_{\beta}=\left\{\beta\left[\beta_{j} \mid k\right], 1 \leq j \leq d, k \notin \beta\right\}$ via Theorem 1 and assuming $(\beta)=1, \Delta\left[k \mid \beta_{j}\right]:=0$ for $k \notin \Delta$, we have

$$
\frac{\partial \Delta}{\partial\left(\beta\left[\beta_{j} \mid k\right]\right)}=\Delta\left[k \mid \beta_{j}\right]
$$

Example 4.1. With the matrix $V$ from Example 3.1, we have with $\Delta=(246)$, $j=2, k=4$ :

$\frac{\partial(246)}{\partial((235)[3 \mid 4])}=\partial\left|\begin{array}{ccc}1 & 0 & 0 \\ v_{41} & v_{42} & v_{43} \\ v_{61} & v_{62} & v_{63}\end{array}\right| / \partial v_{42}=v_{63}=\left|\begin{array}{ccc}1 & 0 & 0 \\ 0 & 1 & 0 \\ v_{61} & v_{62} & v_{63}\end{array}\right|=(236)=(246)[4 \mid 3]$.

Furthermore, the above derivation rule yields

$$
\frac{\partial \Delta}{\partial\left(\beta\left[\beta_{j} \mid k\right]\right)}=0
$$

if the variable $\beta\left[\beta_{j} \mid k\right]$ does not occur in the determinant $\Delta$.

Proof of Theorem 2. $\beta\left[\beta_{i} \mid k\right]$ occurs in $\Delta$ if and only if $k \in \Delta$ and $b_{i} \notin \Delta$. If this is not the case $\Delta\left[k \mid \beta_{i}\right]$ equals 0 . For $k \in \Delta, b_{i} \notin \Delta$ we use the quadratic GrassmannPlücker relation

$$
(\Delta)=(\Delta)(\beta)=\sum_{j=1}^{d} \beta\left[\beta_{i} \mid \Delta_{j}\right] \cdot \Delta\left[\Delta_{j} \mid \beta_{i}\right],
$$

compare $[8]$. Hence $\partial(\Delta) / \partial\left(\beta\left[\beta_{i} \mid k\right]\right)=\Delta\left[k \mid \beta_{i}\right]$.

To eliminate a variable $v \in V_{\beta}$ from the system as described in Section 3 we have to decide which $\Delta \in \Lambda(n, d)$ gives rise to an upper bound (resp. lower bound) for $v$.

It is

$$
\begin{array}{cl} 
& v>-R /\left(\frac{\partial \Delta}{\partial v}\right), \\
\Leftrightarrow \quad & \left(\Delta=v \cdot \frac{\partial \Delta}{\partial v}+R>0 \text { and } \frac{\partial \Delta}{\partial v}>0\right), \\
& \text { or } \quad\left(\Delta=v \cdot \frac{\partial \Delta}{\partial v}+R<0 \text { and } \frac{\partial \Delta}{\partial v}<0\right), \\
\Leftrightarrow \quad & \operatorname{sign}(\Delta) \cdot \operatorname{sign}(\partial \Delta / \partial v)=+1,
\end{array}
$$


and

$$
\begin{gathered}
v<-R /\left(\frac{\partial \Delta}{\partial v}\right) \\
\Leftrightarrow \operatorname{sign}(\Delta) \cdot \operatorname{sign}\left(\frac{\partial \Delta}{\partial v}\right)=-1 .
\end{gathered}
$$

Hence given a $d$-chirotope $\chi$ with $n$ vertices we define for every $v \in V_{\beta}$ the sets of upper and lower bounds as follows:

$$
\begin{aligned}
& \mathcal{U}(v):=\left\{\Delta \in \Lambda(n, d): \chi(\Delta) \cdot \chi\left(\frac{\partial \Delta}{\partial v}\right)=-1\right\}, \\
& \mathscr{L}(v):=\left\{\Delta \in \Lambda(n, d): \chi(\Delta) \cdot \chi\left(\frac{\partial \Delta}{\partial v}\right)=+1\right\} .
\end{aligned}
$$

Here $\Delta, v, \partial \Delta / \partial v \in \Lambda(n, d)$ according to Theorem 2 .

Since $\chi$ was assumed to be simplicial, $\mathscr{U}(v) \cup \mathscr{L}(v)$ is a disjoint union of all determinants containing the variable $v$. A pair $(A, B)$ of disjoint subsets $A, B \subset$ $\Lambda(n, d)$ will be called bipartite if $|a \cap b|=d-1$ for all $a \in A, b \in B$. Especially, $(A, B)$ is bipartite if $A=\varnothing$ or $B=\varnothing$.

\section{Algorithm 4.2.}

Input: Simplicial $d$-chirotope $\chi$ with $n$ vertices.

Output: Solvability sequence $v_{1}, \ldots, v_{d(n-d)}$ according to a basis $\beta \in \Lambda(n, d)$, if it exists.

Remark: The exact definition of a solvability sequence and a proof of the correctness of this algorithm will be given in the next chapter.

0. For all $\beta \in \Lambda(n, d)$

1. Initialize $V:=V_{\beta}, \mathscr{D}:=\Lambda(n, d) ; i:=(n-d) d$

2. If $V=\varnothing$ : Stop. $v_{1}, \ldots, v_{(n-d) d}$ is a solvability sequence.

3. Else:

3.1. Is there a $v \in V$ and a minimal system $R \subset \Lambda(n, d)$ such that $(\mathscr{D} \cap R \cap \mathscr{L}(v), \mathscr{D} \cap R \cap \mathscr{U}(v))$ is bipartite?

\subsection{If yes:}

3.2.1. $v_{i}:=v ; V:=\bigvee\left\{v_{i}\right\}$

3.2.2. $\mathscr{D}:=\mathscr{D} \backslash\left(\mathscr{L}\left(v_{i}\right) \cup \mathscr{U}\left(v_{i}\right)\right)$

3.2.3. $i:=i-1$

\subsubsection{Go to 2 .}

Given a basis $\beta \in \Lambda(n, d)$ and a variable $v=(\beta)\left[\beta_{i} \mid j\right]$, what does it mean geometrically that $(R \cap \mathscr{L}(v), R \cap \mathcal{U}(v))$ is bipartite for a minimal system $R \subset \Lambda(n, d)$ ?

Let there be given a hyperplane $\langle\lambda\rangle, \lambda \in \Lambda(n, d-1)$ and $\beta_{i}, j \notin \lambda$. 


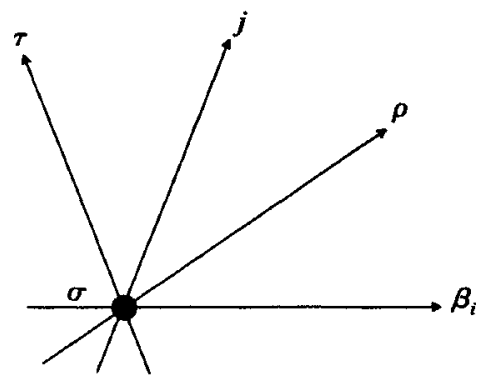

Fig. 2

$\langle\lambda\rangle$ separates $\beta_{i}$ and $j \Leftrightarrow \chi((\lambda, j)) \cdot \chi\left(\left(\lambda, \beta_{i}\right)\right)=-1$,

$$
\begin{aligned}
& \Leftrightarrow \quad x((\lambda, j)) \cdot x\left(\frac{\partial(\lambda, j)}{\partial v}\right)=-1, \\
& \Leftrightarrow \quad(\lambda, j) \in \mathscr{U}(v) .
\end{aligned}
$$

In this sense $\mathscr{U}(v)$ represents the separating hyperplanes of $j$ and $\beta_{i}$ whereas $\mathscr{L}(v)$ represents the nonseparating hyperplanes.

The pair $\left(j, \beta_{i}\right)$ is a contravariant (resp. covariant) pair in the terminology of Cordovil and Duchet [10] if and only if $\mathscr{U}(v)=\varnothing($ resp. $\mathscr{L}(v)=\varnothing)$, a special case of bipartiteness that leads to the notion of max-realizability in the next section.

Let $\left(\sigma_{1}, \ldots, \sigma_{d-2}, \rho, j\right) \in \mathscr{U}(v)$ and $\left(\sigma_{1}, \ldots, \sigma_{d-2}, \tau, j\right) \in \mathscr{L}(v)$.

Clearly, a chirotope $\Pi$ with

$$
\begin{aligned}
\Pi(\lambda) & =\Xi(\lambda) \quad \text { if } \lambda \in \Lambda(n, d) \backslash(\mathcal{U}(v) \cup \mathscr{L}(v)), \\
\Pi(\sigma, \rho, j) & =-\chi(\sigma, \rho, j), \\
\Pi(\sigma, \tau, j) & =-\chi(\sigma, \tau, j),
\end{aligned}
$$

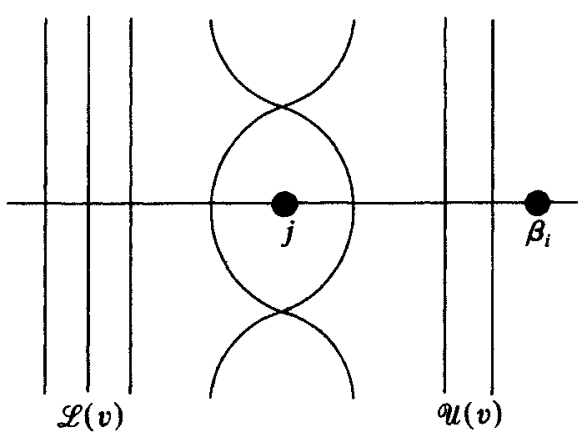

Fig. 3 
cannot exist because of the obvious contradiction to the chirotope definition in the hyperline image $\Pi / \sigma$.

Hence, if $(R \cap \mathscr{L}(v), R \cap \mathcal{U}(v))$ is bipartite, the interval of $j$ on the line $\overline{j \beta_{i}}$ is nonempty in every realization of the restriction of $\chi$ to $\Lambda(n, d) \backslash(U(v) \cup \mathscr{L}(v))$. Emptiness of this interval contradicts the chirotope property by the above argument.

\section{Solvability Sequences}

Given a simplicial $d$-chirotope $\chi$ with $n$ vertices and a basis $\beta \in \Lambda(n, d)$ and an ordering $\left(v_{1}, \ldots, v_{d(n-d)}\right)$ of the set of variables $V_{\beta}:=\left\{v_{1}, \ldots, v_{d(n-d)}\right\}$, we denote

$$
\Lambda_{i}:=\left\{\Delta \in \Lambda(n, d): \frac{\partial \Delta}{\partial v_{j}}=0 \text { for } j>i\right\} .
$$

The sequence $\left(v_{1}, \ldots, v_{d(n-d)}\right)$ is called a solvability sequence for $\chi$ if the following condition holds.

For all $\eta_{1}, \ldots, \eta_{i-1} \in R$ with

$$
\operatorname{sign} \Lambda_{i}\left(\eta_{1}, \ldots, \eta_{i-1}\right)=\left.\chi\right|_{\Lambda_{i-1}} \text {, }
$$

there exists an $\eta_{i} \in R$ with

$$
\operatorname{sign} \Lambda_{i}\left(\eta_{1}, \ldots, \eta_{i}\right)=\left.\chi\right|_{\Lambda_{i}} \text {. }
$$

In other words, every $\eta_{i}$ can be chosen by simply considering those determinants that do not contain $\eta_{i+1}, \ldots, \eta_{d(n-d)}$. This "nonprospective" choice of variables never ends in a blind alley in the case of a solvability sequence.

Remark. A chirotope that has a solvability sequence is realizable.

Theorem 3. Algorithm 4.2 is correct, i.e., any generated sequence $v_{1}, \ldots, v_{d(n-d)}$ is a solvability sequence.

Proof. Let $\eta_{1}, \ldots, \eta_{i-1} \in R$ such that $\operatorname{sign} \Lambda_{i}\left(\eta_{1}, \ldots, \eta_{d(n-d)}\right)=\left.\chi\right|_{\Lambda_{i-1}}$ and $\Delta_{1} \in$ $\Lambda_{i} \cap \mathscr{L}\left(v_{i}\right), \Delta_{2} \in \Lambda_{i} \cap \mathscr{U}\left(v_{i}\right), v_{i}=\beta\left[\beta_{k} \mid j\right]$. Because of the bipartiteness we can assume that $\Delta_{1}=\left(\sigma_{1}, \ldots, \sigma_{d-2}, \rho, j\right)$ and $\Delta_{2}=\left(\sigma_{1}, \ldots, \sigma_{d-2}, \tau, j\right)$. Hence

$$
\begin{aligned}
{\left[\Delta_{2} \cdot \frac{\partial \Delta_{1}}{\partial v_{i}}-\Delta_{1} \cdot \frac{\partial \Delta_{2}}{\partial v_{i}}\right]\left(\ldots, \eta_{l}, \ldots\right) } & =\left[(\sigma \tau j) \cdot\left(\sigma \rho \beta_{k}\right)-(\sigma \rho j) \cdot\left(\sigma \tau \beta_{k}\right)\right]\left(\ldots, \eta_{l}, \ldots\right) \\
& =\left[(\sigma \tau p) \cdot\left(\sigma j \beta_{k}\right)\right]\left(\ldots, \eta_{l}, \ldots\right) \\
& >0
\end{aligned}
$$


by the Grassmann-Plücker relations (compare also Example 3.3) which means that an appropriate $\eta_{i} \in R$ with

$$
\left[R_{1} / \frac{\partial \Delta_{1}}{\partial v_{i}}\right]\left(\eta_{1}, \ldots, \eta_{i-1}\right)<\eta_{i}<\left[R_{2} / \frac{\partial \Delta_{2}}{\partial v_{i}}\right]\left(\eta_{1}, \ldots, \eta_{i-1}\right)
$$

can be found. Since this holds for all $i=1, \ldots, d(n-d)$ the sequence $\left(v_{1}, \ldots, v_{d(n-d)}\right)$ is a solvability sequence.

The fact that Algorithm 4.2 finds a solvability sequence for a sufficiently large class of realizable chirotopes can be established by three arguments:

The max-realizable chirotopes, as described below, already form an extensive subclass having solvability sequences.

Several realizability-compatible point extension techniques from oriented matroid theory and convexity like principal extensions [14], stellar subdivisions and their derivatives are special cases of solvability sequences.

A huge class of practical problems from polyhedral combinatorics has been solved by the above methods [4], [5], [7], [9].

Consider the following addition and multiplication among the integers

$$
\begin{aligned}
& r \oplus s:=\max \{|r|,|s|\} \cdot \operatorname{sign}(r+s), \\
& r \otimes s:=\max \{|r|,|s|\} \cdot \operatorname{sign}(r \cdot s) .
\end{aligned}
$$

Given an integer $n \times d$-matrix $A=\left(a_{i j}\right)$ such that for a fixed $\beta \in \Lambda(n, d)$ the $d \times d$-submatrix with row indices $\beta_{1}, \ldots, \beta_{d}$ is the $d \times d$-unit matrix and that all other entries $a_{i, j}, i \notin \beta$, have pairwise distinct absolute values. Under these conditions the $d$-vector $\widehat{\bigwedge}_{d} A$ according to the above operations $\oplus$ and $\otimes$ is well defined. A chirotope $\chi$ with $\chi=\operatorname{sign} \nwarrow_{d} A$ for such an integer matrix $A$ will be called max-realizable.

Example 5.1. The matrix

$$
A=\left(\begin{array}{rrr}
1 & 0 & 0 \\
0 & 1 & 0 \\
0 & 0 & 1 \\
9 & -5 & 1 \\
10 & -6 & 2 \\
11 & -7 & 3 \\
12 & -8 & 4
\end{array}\right)
$$

induces the cyclic 3 -chirotope with seven vertices $\chi^{7,3}=\operatorname{sign} \pi_{d} A=+1$ which is therefore max-realizable. It can be easily seen that all cyclic chirotopes, i.e., alternating oriented matroids, are max-realizable. 
Theorem 4. For every max-realizable d-chirotope $\chi$ with $n$ vertices, Algorithm 4.2 generates a solvability sequence. Hence $\chi$ is realizable in the ordinary sense.

Proof. Let $\chi:=\operatorname{sign} \bar{\bigwedge}_{d} A$ where $\beta \in \Lambda(n, d)$ is the corresponding basis. We order the variable set $V_{\beta}=\left\{v_{1}, \ldots, v_{d(n-d)}\right\}$ with respect to increasing absolute values of the corresponding entries in the matrix $A$. Then the max-realizability of $\chi$ means that in the notation of the proof of Theorem 3:

$$
\begin{aligned}
& \chi\left(v_{i}\right)=+1 \Rightarrow \Lambda_{i} \cap \mathcal{U}\left(v_{i}\right)=\varnothing \quad \text { and } \\
& \chi\left(v_{i}\right)=-1 \Rightarrow \Lambda_{i} \cap \mathscr{L}\left(v_{i}\right)=\varnothing .
\end{aligned}
$$

Therefore Algorithm 4.2, using the basis $\beta$, finds a trivial bipartite partition in every step.

Let $\chi$ be a $d$-chirotope with $n-1$ vertices. A chirotope $\chi^{\prime}$ with $n$ vertices is called a principal extension of $\chi$ if

$$
\left.\chi^{\prime}\right|_{\Lambda(n, d-1)}=\chi,
$$

and for a basis $\beta \in \Lambda(n, d)$ and some ordering of variables $\left\{v_{1}, \ldots, v_{d(n-d)}\right\}$ with

$$
\left\{v_{d(n-d-1)+1}, \ldots, v_{d(n-d)}\right\}=\left\{\beta\left[\beta_{i} \mid n\right], i=1, \ldots, d\right\},
$$

we have

$$
\mathscr{L}\left(v_{j}\right)=\varnothing \quad \text { or } \quad U\left(v_{j}\right)=\varnothing \quad \text { for } j=d(n-d-1)+1, \ldots, d(n-d) .
$$

This definition is a reformulation of the definition of Las Vergnas [14] or Billera and Munson [2] for oriented matroids. Clearly, $\chi^{\prime}$ is realizable if and only if $\chi$ is realizable. This type of problem reduction will be carried out by Algorithm 4.2 , too. In that sense, inductive principal extensions of the simplex are special cases of max-realizable chirotopes, indicating the richness of this class.

Furthermore, let us mention that several standard extension techniques from convexity like stellar subdivisions [11], pulling the vertices or the concept of strong coverability [1] can be expressed in terms of solvability sequences as well.

Problem. Does there exist a realizable chirotope $\chi$ without solvability sequence?

Although it seems likely that those configurations do exist, no example is known to the authors. ${ }^{2}$

If Algorithm 4.2 has found a solvability sequence, the realizability of the corresponding problem can be proved in the most constructive way, namely, by exhibiting coordinates. Nonrealizability proofs, however, need more efforts and it is not possible to construct obvious contradictions with the described algorithm. Nevertheless the problem reduction by passing over to a minimal system and deleting at least some variables can be very helpful for nonrealizability proofs, too, compare [6].

\footnotetext{
${ }^{2}$ In the meantime Jürgen Richter from Darmstadt gave an example with 13 points in the plane.
} 


\section{The Isotopy Property}

The isotopy problem, posed again by Goodman and Pollack in 1984 [18], deals with a very natural question concerning point configurations in euclidean space. Although it is still open for the general case, a proof for the isotopy property of configurations with solvability sequence will be given in this chapter, showing an interesting connection between realizability and isotopy problems.

Let $M(n \times d, R)$ denote the topological space of all real $n \times d$-matrices with the canonical topology. The map $\wedge_{d}$ is an imbedding of $M(n \times d, R)$ in the $d$ th exterior power $\bigwedge_{d} R^{n}$ of $R^{n}$. For every chirotope $\chi$ let

$$
\sigma_{\chi}:=\left\{\Xi \in \bigwedge_{d} R^{n}, \text { sign } \Xi=\chi\right\}
$$

Conjecture 6.1 (Isotopy Problem) [18]. For every simplicial chirotope $\chi$ the topological space $\bigwedge_{d} M(n \times d, R) \cap \hat{\sigma}_{\chi}$, also called realization space of $\chi$, is path connected.

In other words, two equivalent nondegenerate point configurations can always be deformed into each other in a continuous way whilst keeping the corresponding chirotope.

Theorem 5. For every simplicial chirotope $\chi$ with solvability sequence the space $\bigwedge_{d} M(n \times d, R) \cap \mathcal{O}_{\chi}$ is contractible, hence path connected.

Proof. We shall construct a homeomorphism

$$
\mathscr{H}:(0,1)^{d(n-d)} \rightarrow \bigwedge_{d} M(n \times d, R) \cap \mathcal{O}_{\chi}
$$

Let $v_{1}, \ldots, v_{d(n-d)}$ be a solvability seuqence of $\chi$ according to the basis $\beta \in \Lambda(n, d)$ and $\Lambda_{i}, \mathscr{L}\left(v_{i}\right), \mathcal{U}\left(v_{i}\right) \subset \Lambda(n, d)$ as before. Every $\Delta \in \Lambda_{i}$ will be interpreted as a homogeneous polynomial $\Delta\left(v_{1}, \ldots, v_{i}\right)$. We define continuous functions $f_{i}$ and $g_{i}$ in $i-1$ variables.

$$
\begin{aligned}
& f_{i}\left(v_{1}, \ldots, v_{i-1}\right):=\min \left\{-\Delta\left(v_{1}, \ldots, v_{i-1}, 0\right) / \frac{\partial \Delta}{\partial v_{i}}\left(v_{1}, \ldots, v_{i-1}\right): \Delta \in \mathscr{U}\left(v_{i}\right)\right\}, \\
& g_{i}\left(v_{1}, \ldots, v_{i-1}\right):=\max \left\{-\Delta\left(v_{1}, \ldots, v_{i-1}, 0\right) / \frac{\partial \Delta}{\partial v_{i}}\left(v_{1}, \ldots, v_{i-1}\right): \Delta \in \mathscr{L}\left(v_{i}\right)\right\},
\end{aligned}
$$

which describe the upper and lower bound for $v_{i}$ if the $v_{k}, k<i$, are already chosen. Since we have a solvability sequence, $f_{i}>g_{i}$ whenever $v_{1}, \ldots, v_{i-1}$ realizes $\left.\chi\right|_{\Lambda_{i-1}}$.

Let $h(t ; a, b)$ for $a, b \in R \cup\{-\infty, \infty\}, t \in(0,1)$ be a real-valued continuous function such that

$$
\lim _{t \rightarrow 0} h(t ; a, b)=a \text { and } \lim _{t \rightarrow 1} h(t ; a, b)=b
$$


Using this function we can construct the desired homeomorphism between $(0,1)^{d(n-d)}$ and the realization space of $\chi$ as

$$
\begin{aligned}
& \mathscr{H}:(0,1)^{d(n-d)} \rightarrow \Lambda_{d} M(n \times d) \cap \mathcal{O}_{x}, \\
& \left(t_{1}, \ldots, t_{d(n-d)}\right) \mapsto \Lambda(n, d)\left(v_{1}, \ldots, v_{d(n-d)}\right),
\end{aligned}
$$

with

$$
v_{i}:=h\left(t_{i} ; g_{i}\left(v_{1}, \ldots, v_{d(n-d)}, f_{i}\left(v_{1}, \ldots, v_{d(n-d)}\right)\right)\right.
$$

Note that if we consider $\mathscr{H}$ as a mapping $\mathscr{H}:(0,1)^{d(n-d)} \rightarrow \bigwedge_{d} M(n \times d, R), \mathscr{H}$ is a homeomorphism onto its image Im $\mathscr{H}$ for every chirotope $\chi$. If $v_{1}, \ldots, v_{d(n-d)}$ fails to be a solvability sequence, however, $\operatorname{Im} \mathscr{H}$ will exceed $O_{x}$.

\section{References}

1. A. Altshuler and I. Shemer, Construction theorems for polytopes, Israel J. Math. 47 (1984), 99-100.

2. L. J. Billera and B. S. Munson, Polarity and inner products in oriented matroids, Preprint, Cornell University, Ithaca, 1983.

3. R. G. Bland and M. Las Vergnas, Orientability of matroids, J. Combin. Theory Ser. B 24 (1978), 94-123.

4. J. Bokowski and U. Brehm, A new polyhedron of genus 3 with 10 vertices, Colloq. Math. Soe. János Bolyai, to appear.

5. J. Bokowski, G. Ewald and P. Kleinschmidt, On combinatorial and aftine automorphisms of polytopes, Israel J. Math. 47 (1984), 123-130.

6. J. Bokowski and K. Garms, Altshuler's sphere $M_{425}^{10}$ is not polytopal, European $J$. Combin., to appear.

7. J. Bokowski and I. Shemer, Neighborly 6-polytopes with 10 vertices, Israel J. Math., submitted.

8. J. Bokowski and B. Sturmfels, Oriented matroids and chirotopes-mroblems of geometric realizability, Preprint, TH, Darmstadt, 1985.

9. J. Bokowski and B. Sturmfels, Polytopal and non-polytopal spheres-An algorithmic approach, Preprint, TH, Darmstadt, 1985.

10. R. Cordovil and P. Duchet, On the number of sign-invariant points in oriented matroids, Preprint, Lisboa, 1985.

11. G. Ewald, P. Kleinschmidt, U. Pachner and C. Schulz, Neuere Entwicklungen in der kombinatorischen Konvexgeometrie, in Contributions to Geometry (J. Tölke and J. M. Wills eds.) (Proceedings of the Geometry-Symposium, Siegen, June, 1978), Birkhäuser, Basel,

12. J. E. Goodman and R. Pollack, Upper bounds for configurations and polytopes in $R^{d}$, Discrete Comput. Geom, to appear.

13. B. Grüibaum, Convex Polytopes, Interscience, London, 1967.

14. M. Las Vergnas, Extensions ponctuelles d'une géométrie combinatoire orientée. Colloque C.N.R.S. Problemes combinatoires et théorie des graphes, Orsay 1976, Paris, 1978.

15. M. Las Vergnas, Matroides orientables, Preprint, April 1974; announced in C. R. Acad. Sci. Paris 280 (January 20, 1975).

16. M. Las Vergnas, Bases in oriented matroids, J. Combin. Theory Ser. B 25 (1978), 283-289.

17. J. Lawrence, Oriented matroids and multiple ordered sets, Linear Algebra Appl. 48 (1982), 1-12.

18. Tagungsbericht Oberwolfach, Tagung über kombinatorische Geometrie, September 1984, List of problems.

19. B. Sturmfels, Zur linearen Realisierbarkeit orientierter Matroide. Diplomarbeit, Darmstadt, 1985.

20. A. Tarski, A Decision Method for Elementary Algebra and Geometry. Berkeley and Los Angeles, 1951.

Received July 11, 1985, and in revised form July 21, 1986. 